\title{
Actual trends in mass spectrometry of glycosaminoglycans
}

\author{
Adrian Cristian Robu ${ }^{1}$, Laurentiu Popescu ${ }^{1,2}$, Alina Diana Zamfir ${ }^{1,3, *}$ \\ ${ }^{1}$ National Institute for Research and Development in Electrochemistry and Condensed Matter, \\ RO-300224, Timisoara, Romania \\ ${ }^{2}$ Faculty of Physics, West University of Timisoara, RO-300223, Timisoara, Romania \\ ${ }^{3}$ Aurel Vlaicu University of Arad, RO-310130, Arad, Romania
}

(Received 20 March, 2017; accepted 25 April, 2017)

\begin{abstract}
During the past few years, in the field of glycosaminoglycomics, mass spectrometry (MS) emerged as one of the most efficient analytical techniques due to its valuable contribution to compositional and structural determination of various glycosaminoglycan (GAG) classes. This review highlights briefly some of the most modern and efficient MS approaches and allied separation techniques such as liquid chromatography, ion mobility and capillary electrophoresis designed to offer high performance analysis at elevated sensitivity, with drastic reduction of the sample consumption and processing prior to MS. Such bioanalytical platforms are able to provide valuable and straightforward information on the GAG oligosaccharide domains and their structure in terms of epimerization, composition of the disaccharide repeats and their sulfation status. The state-of-the-art in the field of the MS techniques applied to GAG analyses is focused here particularly on chondroitin and dermatan sulfate GAGs extracted and purified from the extracellular matrix and cerebral tissue.
\end{abstract}

Keywords: glycosaminoglycans, electrospray ionization, high resolution mass spectrometry, screening, fragmentation analysis

\begin{abstract}
Abbreviations: $\mathrm{CE}=$ capillary electrophoresis; $\mathrm{CS}=$ chondroitin sulfate; DS=dermatan sulfate; ECM=extracellular matrix; EDD=electron detachment dissociation; ESI=electrospray ionization; FAIMS=high-field asymmetric waveform ion mobility spectrometry; FTICR=Fourier transform ion cyclotron resonance; FTMS=Fourier transform mass spectrometry; GAG=glycosaminoglycan; GalNAc=N-acetylgalactosamine; GlcA=glucuronic acid; HexA=hexuronic acid; HILIC=hydrophilic interaction chromatography; HS=heparan sulfate; IdoA=iduronic acid; IMS=ion mobility spectrometry; $\mathrm{KS}=$ keratan sulfate; LC=liquid chromatography; MALDI=matrix assisted laser desorption/ionization; MS/MS=tandem mass spectrometry; $\mathrm{MW}=$ molecular weight; $\mathrm{NETD}=$ negative electron transfer dissociation; $\mathrm{PG}=$ proteoglycan; $\mathrm{RF}=$ radio fire; $\mathrm{SEC}=$ size-exclusion chromatography; $\mathrm{SPE}=$ solid phase extraction; $\mathrm{UA}=$ uronic acid
\end{abstract}

\section{INTRODUCTION}

Glycosaminoglycans (GAGs), the major components of the extracellular matrix (ECM), are O-glycans responsible for a variety of biological activities of the proteoglycans (PGs), playing an essential role in ECM organization, maturation, various pathological and physiological processes, tissue development or cellular behavior (1-3). An interesting aspect related to the PGs is that GAGs influence not only the biological activities, but also their properties, due to the fact that, unlike the $N$-glycans decorating PGs, GAGs represent over a half of the PGs molecular weight (MW) (4). For a better understanding of the role played by GAGs in various pathologies, their structural determination is a fundamental requirement (5) and assumes collection of data on the repeating disaccharide unit that constitutes the chain (6).

GAG class is divided in six main sub-classes: chondroitin sulfate (CS), dermatan sulfate (DS), heparin, heparan sulfate (HS), keratan sulfate (KS) and hyaluronic acid; among GAGs, CS and DS sub-classes are $O$-covalently attached to a wide diversity of PG proteins via a tetrasaccharide sequence, having the composition GlcA-Gal-Gal-Xyl, known as the linkage region, linkage bridge or simply linker (7). CS species exhibit

*Corresponding author (Tel/Fax: +40-256-494413; E-mail: alina.zamfir@uav.ro) 


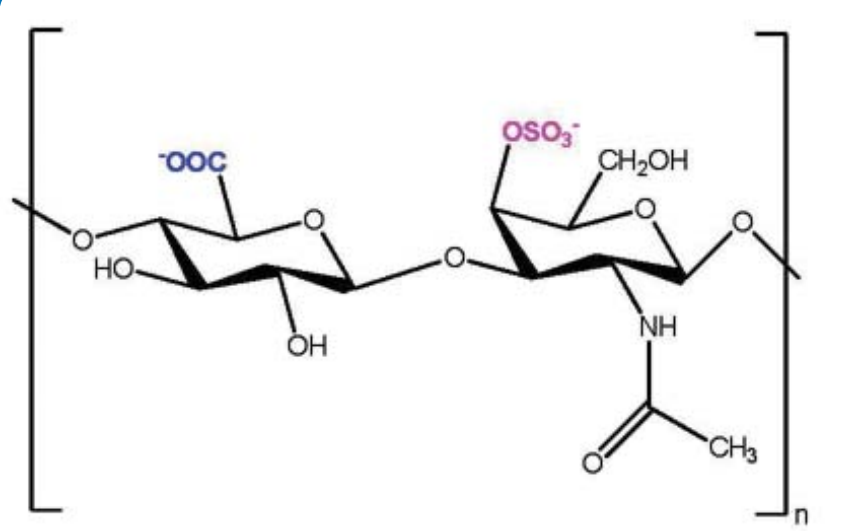

Chondroitin 4-sulfate

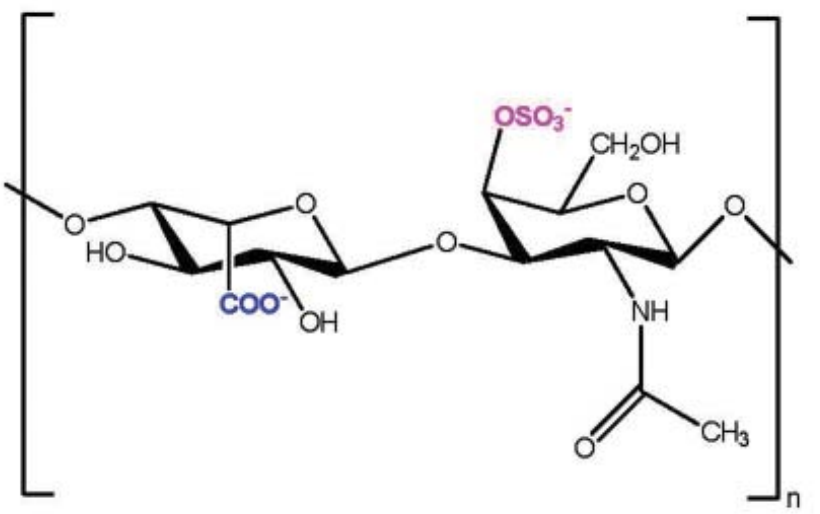

Dermatan sulfate

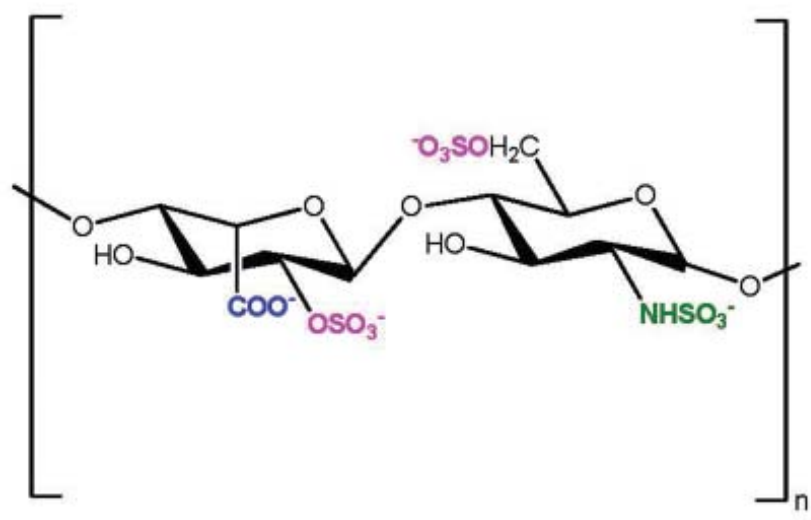

Heparin

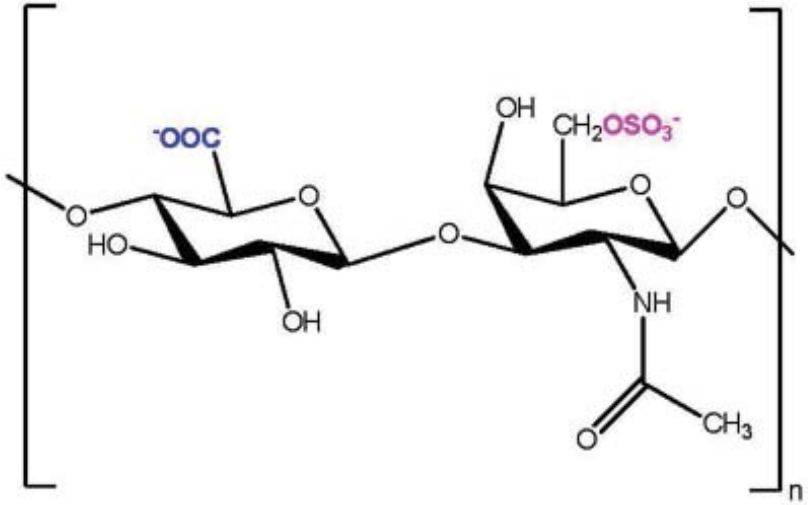

Chondroitin 6-sulfate

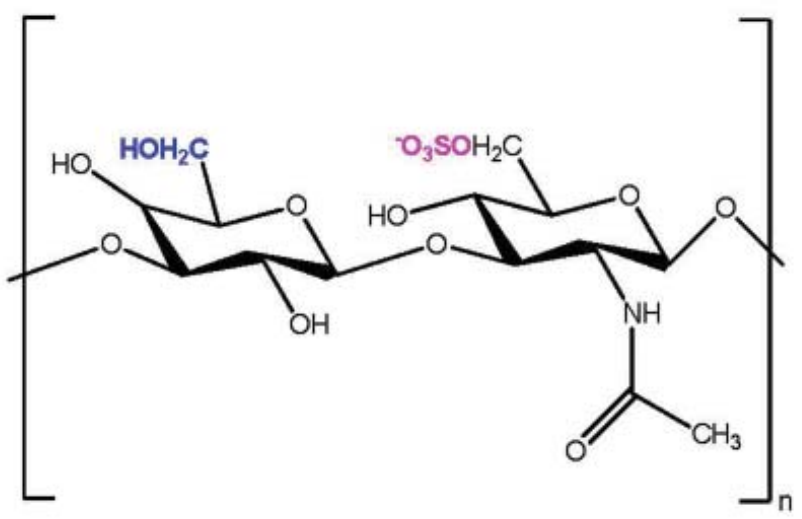

Keratan sulfate

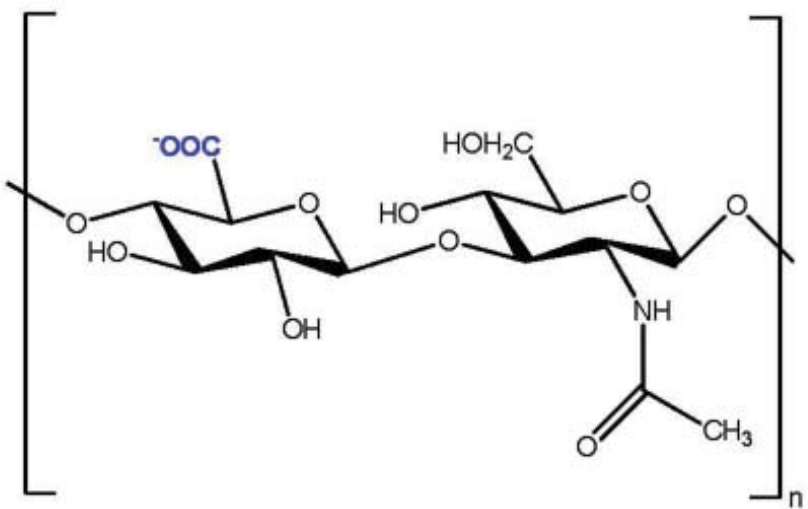

Hyaluronic acid

Fig. 1. - The general structure of glycosaminoglycans. Reprinted and adapted with permission from (4).

similar structure to DS, the difference between the two compounds being given by the nature of the hexuronic acid (HexA) within the repeating disaccharide unit: D-glucuronic acid for CS (GlcA) and L-iduronic acid (IdoA), or its isomeric form, GlcA for DS. CS and DS are often found in hybrid forms in which DS is interspersed among CS motifs. Besides their general structure (Fig. 1), the GAGs possess an essential characteristic, such as the degree of sulfation, influencing their biological properties (8). Within this process, the oligosaccharide part is modified at the $N$-acetylgalactosamine (GalNAc) residue, whereas a small amount of the uronic acid (UA) might be sulfated in position 2 , if an additional sulfate group exists (9).

In this paper, we review the actual trends in mass spectrometry of GAGs by employing some of the most 
powerful techniques which can ease, improve and speed the GAG analyses. The methods described here are able to provide data with an excellent accuracy and high sensitivity, thus opening new perspectives in the fields.

\section{MODERN WORKFLOW FOR GAG ANALYSIS}

The main steps to be performed prior to the MS analysis of GAGs (Fig. 2) encompass (10): i) release of CS/DS chains through a $\beta$-elimination reaction, which does not depend on the sulfation content and pattern; ii) digestion of CS/DS chains using chondroitin $\mathrm{AC}$ and chondroitin $\mathrm{B}$ lyases; iii) size fractionation by gel filtration. Chondroitin B lyase cleaves the linkage between GalNAc and L-IdoA, whereas chondroitin AC I lyase cleaves the linkage between GalNAc and D-GlcA; the cleavages are induced by the neutralization of the negative charge of the carboxylic acid group, followed by abstraction of the C5 proton (chiral center) and subsequent elimination of the C4 hydroxyl group by introducing a C4-C5 double bond. According to the block diagram in Fig. 2, the last step in GAG analysis consists of the ESI MS ${ }^{\mathrm{n}}$ experiments, which target the determination of the molecular ion masses, revealing their composition and the overall extent

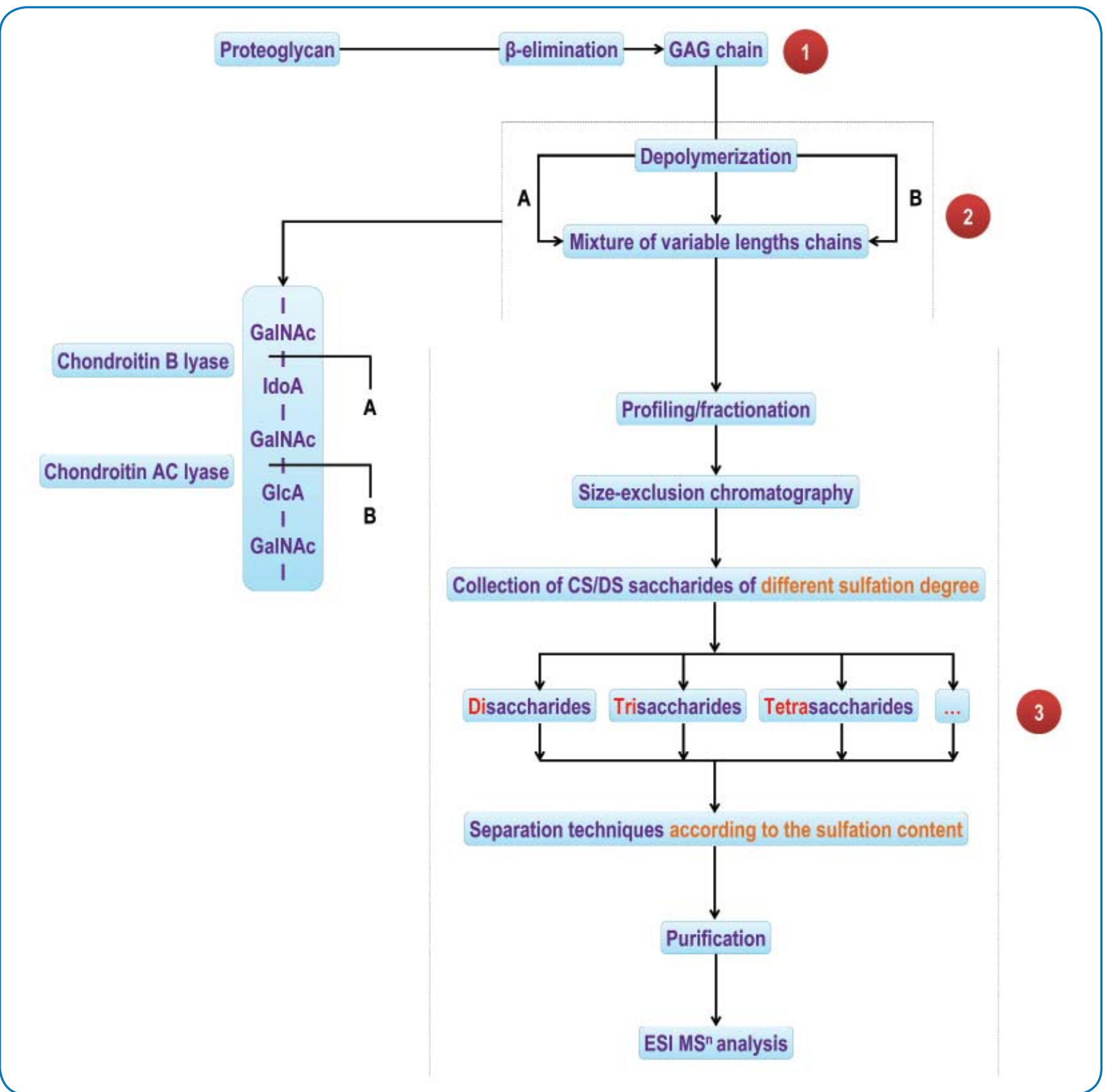

Fig. 1. - GAG analysis block diagram based on the recognition specificity of chondroitin lyases and MS. The GAG chains released from proteoglycans by $\beta$-elimination are depolymerized with chondroitin lyases, based on their recognition specificity. The variable length chain mixture obtained is further subjected to fractionation and separation by SEC. Before the ESI MS ${ }^{\mathrm{n}}$ analysis, the polled CS/DS fractions are further separated, according to their sulfation degree and purified. Reprinted and adapted with permission from (18). 
of sulfation followed by a possible correlation of unusual sulfation content with HexA-epimerization and also, the identification of the sulfate group positions along the chain using the masses of the fragment ions generated by multistage $\mathrm{MS}^{\mathrm{n}}$.

\section{METHODS FOR GAG SEPARATION PRIOR TO MS}

The analysis of GAG oligosaccharide raw mixtures by MS might encounter several problems caused by: i) the in-source fragmentation of the labile sulfate groups; ii) the difficulty to ionize long GAG chains; iii) high complexity of the mass spectrum, due to the formation of ions bearing different charge states; iv) simultaneous ionization and detection of chains exhibiting a variable sulfation degree; v) the formation of isobaric, possibly overlapped ions, capable to jeopardize the assignment to the real oligosaccharide structure, due to the complexity of the mixtures; vi) the analysis of heterogeneous GAG samples in which long and short, under-, regular and oversulfated or even nonsulfated chains may be concomitantly present, and need to be distinguished. Hence, a proficient separation that allows the molecular mass determination of individual components and subsequent fragmentation analysis in tandem MS (MS/MS) experiments for a detailed structure analysis is required.

With respect to the separation process, one of the most challenging topics nowadays is related to the development of ion mobility mass spectrometry (IMS MS). Recently, the analyses performed on heparin octasaccharides by Seo et al., 2012 (11) have indicated that IMS MS is able to identify and separate isomers based on their structural conformations. In this work, the authors were able to resolve two ion populations within the ion mobility cell, a feature which suggests the presence of two different conformations having the same sulfation pattern.

Liquid chromatography (LC) and rapid-fire (RF) tandem mass spectrometry were shown $(12,13)$ to provide an increased sensitivity and high accuracy for simultaneous measurement of DS, HS or KS. Additionally, the study of Tomatsu et al., 2014 (12), indicated that GAGs having close $m / z$ values can be separated with high accuracy by using LC-MS/MS in conjunction with the RF-MS/MS technique. This method encompasses an integrated solid phase extraction (SPE) robot, hence, the sample processing time is reduced. The conjunction of these methods can therefore provide both high throughput and economic advantages.

For this type of molecules, in addition to these valuable techniques, the researchers in the field have used alternative methods, such as capillary electrophoresis (CE) in conjunction with electrospray ionization (ESI) MS and tandem MS. Some of the goals of CE application for GAG analyses (4) are to associate the sulfation pattern with the epimerization of HexA, separate the GAG species exhibiting a diverse sulfation content and determine by tandem MS the sulfate group(s) position along the oligosaccharide chain and if possible, within the monosaccharide ring. This method allows the separation of species which are electrosprayed from the CE capillary into MS at high sensitivity, offering valuable information on both the active domains of GAGs and their fine structure.

\section{APPROACHING GAGS BY HIGH PERFORMANCE MS TECHNIQUES}

When used together, high-field asymmetric waveform ion mobility spectrometry (FAIMS) and Fourier transform ion cyclotron resonance (FTICR) MS were shown (14) to provide significant information on isomeric and isobaric GAG structures, thus proving the usefulness of this analysis type on GAGs. In the work of Kailemia et al., 2014 (14), the ability to produce spectra with high-information content was achieved by a valuable technique for GAG characterization, such as electron detachment dissociation (EDD), which can be used to determine the site of the sulfate groups attached to the analyzed GAGs, together with FAIMS and FTICR MS, which has shown that isomeric and isobaric GAG gas phase ions having the same mass/charge ratio may be separated.

In the last few years, fully automated chip-based nanoESI systems coupled to different MS instruments demonstrated their potential for a successful mapping and structural characterization of GAGs, due to: i) flexibility; ii) several chemical, biochemical and economical advantages, i.e., the simplification of some laborious biochemical strategies, required in the MS research; iii) automated sample infusion; iv) increased ionization efficiency; v) high quality of spectra; vi) reduction of cross-contamination; vii) possibility for high-throughput experiments; viii) minimization of sample loss and sample handling. Hence, by using chip-based MS it was possible for instance to successfully perform a structural analysis of CS/DS in human decorin (15), identify an unusually sulfated tetrasaccharide motif in the mouse brain (16), or provide information on the essential structural elements such as chain length, epimerization, sulfate content, and sulfation sites in a brain tissue sample (17).

Development of nanoESI Orbitrap MS and CID multistage $M S^{n}$ (9), yielded recently valuable information on the structure of CS/DS chains in brain. Hence, by high resolution measurement, the occurrence of an atypical species presenting a bisulfated CS disaccharide formed by 3-O-sulfate-4,5-D-GlcA and 6-O-sulfate-GalNAc moieties revealing clear differences in the CS/DS expression induced by the postnatal brain development was discovered. In this study, the results have revealed 
for the first time the existence of an age-related structural variability of the CS/DS motifs in the analyzed samples, which corroborated the previous findings related to the incidence of oversulfated motifs in cerebral tissue.

Next to nanoESI, matrix assisted laser desorption/ionization (MALDI) MS technique applied to either fractions containing GAG oligosaccharides or complex mixtures proved its efficiency (18), due to the ability of this method to create mostly singly charged species, in comparison to the multiply charged ions produced by using an ESI source, simplfying the data interpretation. Besides these features, this method presents other advantages, as compared with ESI, such as a much higher tolerance to different contaminants, mostly salts and an elevated applicability to high-throughput experiments. All these findings substantiate the impact of how charge state contributes to gas phase structures and their subsequent measurement.

\section{GAG STRUCTURAL CHARACTERIZATION PLATFORMS}

The current state-of-the-art related not only the GAG mapping, but also their structural characterization using mass spectrometric approaches includes various techniques such as the online hydrophilic interaction chromatography (HILIC) Fourier transform mass spectrometry (FTMS) for the analysis of oligosaccharide fragment ions of low MW heparin (LMWH). This method was applied by $\mathrm{Li}$ et al., 2014 (19), for the analysis of LMWHs resulted from digestion with heparin lyase II. Here, the bottom-up approach, which provided a detailed structural analysis and also, quantitative information was combined with a top-down approach, opening a new direction towards an advanced quality control and quality assurance process on the topic of the low MW heparin production. To distinguish the epimers, in the work of Wolff et al., 2010 (20), the negative electron transfer dissociation (NETD) technique was coupled to an ion trap mass spectrometer for the analysis of GAGs. Thereby, both glycosidic and cross-ring cleavages could be observed, as well as even- and odd-electron products. This method dissociates GAGs producing abundant and useful fragmentation similar to EDD, being able to distinguish between the epimers of HexA in HS tetrasaccharides, in a single tandem MS experiment. By employing these modern platforms, it was possible to identify oligosaccharide species derived from GAG mixtures with different sulfation degrees, making possible their exhaustive characterization. The gathered structural information is often essential not only for understanding the mechanisms of various pathologies at the level of the extracellular matrix but also for rendering a tentative diagnostic based on the discovery of disease associated CS/DS species.

\section{CONCLUSIONS}

In this paper, the most modern developments in the analysis of GAGs using mass spectrometric approaches were highlighted and critically assessed. This review describes not only the general trends in the separation of this type of polysaccharides, a step required for the molecular mass determination of individual components and subsequent fragmentation analysis in tandem MS experiments, but also several efficient approaches for GAG screening using ultra high-resolution MS techniques and structural characterization by sequencing. All these methods allow the identification of species at a high sensitivity, offering valuable information on the active domains of GAGs and their fine structure. Moreover, the findings emphasized here substantiate the impact of the modern MS technology on the progress of the glycosaminoglycomics field. Such powerful techniques are able to significantly increase data reliability and also to provide structural information at the elevated accuracy necessary for elucidating the role played by these particular biopolymers in the extracellular matrix and not only.

Acknowledgements: This work was supported by the Romanian National Authority for Scientific Research, CNCS - UEFISCDI, project PN-II-PT-PCCA-2013-4-0191 to A.D.Z. Conflict of interests: none declared.

\section{REFERENCES}

1. Ghatak S, Maytin EV, Mack JA, Hascall VC, Atanelishvili I, Moreno-Rodriguez R, Markwald RR, Misra S, Roles of Proteoglycans and Glycosaminoglycans in Wound Healing and Fibrosis, Int. J. Cell Biol., 2015, 834-893 (2015).

2. Xiong A, Kundu S, Forsberg-Nilsson K, Heparan sulfate in the regulation of neural differentiation and glioma development, FEBS J., 281, 4993-5008 (2014).

3. Theocharis AD, Gialeli C, Bouris P, Giannopoulou E, Skandalis SS, Aletras AJ, Iozzo RV, Karamanos NK, Cell-matrix interactions:

focus on proteoglycan-proteinase interplay and pharmacological targeting in cancer, FEBS J., 281, 5023-5042 (2014).

4. Zamfir AD, Applications of capillary electrophoresis electrospray ionization mass spectrometry in glycosaminoglycan analysis, Electrophoresis, 37, 973-986 (2016).

5. Zaia J, Glycosaminoglycan Glycomics Using Mass Spectrometry, Mol. Cell. Proteomics, 12, 885-892 (2013).

6. Zamfir A, Seidler DG, Schönherr E, Kresse H, Peter-Katalinić J, On-line sheathless capillary electrophoresis/ nanoelectrospray 
ionization-tandem mass spectrometry for the analysis of glycosaminoglycan oligosaccharides, Electrophoresis, 25, 2010-2016 (2004).

7. Seidler DG, Peter-Katalinić J, Zamfir AD, Galactosaminoglycan function and oligosaccharide structure determination, Sci. World J., 7, 233-241 (2007).

8. Schnabelrauch M, Scharnweber D, Schiller J, Sulfated glycosaminoglycans as promising artificial extracellular matrix components to improve the regeneration of tissues,

Curr. Med. Chem., 20, 2501-2523 (2013).

9. Robu AC, Popescu L, Munteanu CVA, Seidler DG, Zamfir AD, Orbitrap mass spectrometry characterization of hybrid chondroitin/ dermatan sulfate hexasaccharide domains expressed in brain, Anal. Biochem., 485, 122-131 (2015).

10. Zamfir AD, Flangea C, Sisu E, Serb AF, Dinca N, Bruckner P, Seidler DG, Analysis of novel over-and under-sulfated glycosaminoglycan sequences by enzyme cleavage and multiple stage MS, Proteomics, 9, 3435-3444 (2009).

11. Seo Y, Andaya A, Leary JA, Preparation, Separation, and Conformational Analysis of Differentially Sulfated Heparin Octasaccharide Isomers using Ion Mobility Mass Spectrometry, Anal Chem., 84, 2416-2423 (2012).

12. Tomatsu S, Shimada T, Mason RW, Kelly J, LaMarr WA, Yasuda E, et al., Assay for Glycosaminoglycans by Tandem Mass Spectrometry and its Applications, J. Anal. Bioanal. Tech., 2, S2-006 (2014).

13 Shimada T, Kelly J, LaMarr WA, van Vlies N, Yasuda E, Mason RW, et al., Novel heparan sulfate assay by using automated highthroughput mass spectrometry: application to monitoring and screening for mucopolysaccharidoses, Mol. Genet. Metab., 113, 92-99 (2014).
14. Kailemia MJ, Park M, Kaplan DA, Venot A, Boons GJ, Li L, Linhardt RJ, Amster IJ, High-Field Asymmetric-Waveform Ion Mobility Spectrometry and Electron Detachment Dissociation of Isobaric Mixtures of Glycosaminoglycans, J. Am. Soc. Mass. Spectrom., 25, 258-268 (2014).

15. Zamfir AD, Flangea C, Sisu E, Seidler DG, Peter-Katalinić J, Combining size-exclusion chromatography and fully automated chip-based nanoelectrospray quadrupole time-of-flight tandem mass spectrometry for structural analysis of chondroitin/dermatan sulfate in human decorin, Electrophoresis, 32, 1639-1646 (2011).

16. Flangea C, Petrescu AJ, Seidler DG, Munteanu CV, Zamfir AD, Identification of an unusually sulfated tetrasaccharide chondroitin/dermatan motif in mouse brain by combining chip-nanoelectrospray multistage MS2 -MS4 and high resolution MS, Electrophoresis, 34, 1581-1592 (2013).

17. Zamfir AD, Flangea C, Serb A, Sisu E, Zagrean L, Rizzi A, Seidler DG, Brain chondroitin/dermatan sulfate, from cerebral tissue to fine structure: extraction, preparation, and fully automated chip-electrospray mass spectrometric analysis, Meth. Mol. Biol., 836, 145-159 (2012).

18. Sisu E, Flangea C, Serb A, Zamfir AD, Modern developments in mass spectrometry of chondroitin and dermatan sulfate glycosaminoglycans, Amino Acids, 41, 235-256 (2011).

19. Li G, Steppich J, Wang Z, Sun Y, Xue C, Linhardt RJ, Li L, Bottom-Up Low Molecular Weight Heparin Analysis Using Liquid Chromatography-Fourier Transform Mass Spectrometry for Extensive Characterization, Anal. Chem., 86, 6626-6632 (2014).

20. Wolff JJ, Leach FE 3rd, Laremore TN, Kaplan DA, Easterling ML, Linhardt RJ, Amster IJ, Negative Electron Transfer Dissociation of Glycosaminoglycans, Anal. Chem., 82, 3460-3466 (2010). 